\title{
Obesidade infantil no Brasil: uma revisão integrativa
}

Childhood obesity in Brazil: an integrative review

La obesidad infantil en Brasil: uma revisión integradora

Jean Brum Jardim ${ }^{1}$; Inês Leoneza de Souza ${ }^{2}$

\section{Resumo}

A Organização Mundial de Saúde, em 2012 alerta as nações quanto a alarmante e crescente presença da obesidade no mundo e a imprescindibilidade de instituírem-se em caráter de urgência ações de combate a este mal, que correlacionava-se a 2/3 das mortes no mundo. Em 2014, novo alerta é emitido convocando o globo desta vez ao combate à obesidade infantil, a forma consensualmente mais eficaz de enfrentamento à obesidade. O presente estudo tem como objetivo analisar e discutir as publicações acerca da obesidade infantil no Brasil e para tal realizou-se revisão integrativa de 39 artigos selecionados através de busca nas bases de dados da Biblioteca Virtual de Saúde, até janeiro de 2016. Os resultados comprovaram a presença de índices alarmantes de obesidade infantil nas crianças brasileiras, bem como a multiplicidade de causas e efeitos. Houve pouca participação da enfermagem nas pesquisas. Os estudos são predominantemente quantitativos e ocorrem em escolas. Houve pouca inclusão dos familiares e professores como objetos de estudo e mesmo como participantes nestes. Poucos autores propuseram elencar soluções factíveis à prevenção e combate à obesidade. Sugere-se, portanto que novas pesquisas sejam realizadas por enfermeiros, em caráter quanti-qualitativo, envolvendo crianças, familiares e professores cujo objetivo prioritário seja elaborar e testar estratégias de enfrentamento ao assim denominado, mal do século.

Descritores: obesidade infantil; obesidade pediátrica; saúde pública; obesidade; enfermagem pediátrica.

\section{Abstract}

The WHO, in 2012 warns the nations about the alarming and growing presence of obesity in the world and the indispensability of establish an urgency state of actions to combat this evil, which is correlated with two thirds of deaths worldwide. In 2014 a new alert is issued calling upon the globe, this time to combat childhood obesity; consensually the most effective way to face the obesity. This study aims to analyze and discuss the publications about childhood obesity in Brazil and to do so an integrative review of 39 articles selected through search in the Virtual Health Library databases, until January 2016, was conducted. The results show the presence of alarming rates of childhood obesity in Brazilian children, in addition to the multiplicity of its causes and effects. There is few participation of nursing in the research. The studies are predominantly quantitative and occur in schools. There was few inclusion of family members and teachers as objects of study and even as participants on those. Few authors have proposed to list feasible solutions to prevent and combat obesity. It is suggested therefore that further research be carried out by nurses, in quantitative and qualitative stance, involving children, families and teachers whose primary goal is to develop and

\footnotetext{
${ }^{1}$ Graduado em Enfermagem / UFRJ e pós-graduando em Saúde da Família / Universidade Estácio de Sá. Professor do curso técnico de Enfermagem da Escola Técnica José Rodrigues da Silva. End.: Rua das Cabiúnas 332, apt. 401. Nova Aroeiras, Macaé - RJ. CEP: 27946-040. E-mail: jbjenf@ hotmail.com

${ }^{2}$ Doutorado em Enfermagem /EEANUFRJ. Professora Adjunta em Saúde Coletiva do curso de Graduação em Enfermagem. Campus UFRJ-Macaé. End: Praia do Flamengo 186/806. Flamengo -RJ CEP.22220-030. E-mail: ines.leoneza@gmail.com
} 
ISSN 2179-6750

test strategies for facing the so called, evil of the century.

Key-words: childhood obesity; pediatric obesity; public health; obesity; pediatric nursing.

\section{Resumen}

La OMS, en 2012 señala a las naciones cuanto a la alarmante y creciente presencia de la obesidad en el mundo y la imprescindibilidad de instituirse en carácter de urgencia acciones de combate a este mal, que se correlacionaba a 2/3 de las muertes en el mundo. En 2014, una nueva alarma es emitido convocando el globo de esta vez al combate a la obesidad infantil, la forma consensualmente más eficaz de enfrentamiento a la obesidad. El estudio tiene como objetivo analizar y debatir a las publicaciones a cerca de la obesidad infantil en Brasil y para tal se realizó una revisión interactiva de 39 artículos seleccionados a través de buscas en las bases de datos de la Biblioteca Virtual de Salud, hasta enero de 2016. Los resultados comprueban la presencia de índices alarmantes de obesidad infantil en los niños brasileños, así como la multiplicidad de causas y efectos. Hubo poca participación de la enfermería en las investigaciones. Los estudios son predominantemente cuantitativos y ocurren en las escuelas. Hubo poca inclusión de los familiares y profesores, como objetos de estudios y mismo como participantes en estos. Pocos autores propusieron encontrar soluciones factibles a la prevención al combate a la obesidad. Sugiriese, por lo tanto que nuevas investigaciones se han realizadas por enfermeros en carácter cualitativos, envolviendo niños familiares y profesores cuyo objetivo prioritario sea corroborar y testar estrategias de enfrentamiento al así denominado mal del siglo.

Palabras-claves: obesidad Infantil; obesidad pediátrica; salud pública; obesidad; enfermería; enfermería pediátrica.

\section{Introdução}

A partir do relatório "Estatísticas Mundiais de Saúde 2012”, publicado em dezesseis de maio de dois mil e doze pela Organização Mundial de Saúde (OMS), é possível dimensionar a problemática representada pela obesidade e sobrepeso no mundo. Segundo o relatório, a cada ano morrem no mundo 2,8 milhões de pessoas devido ao sobrepeso e à obesidade, o que aumenta o risco de doenças cardiovasculares, acidentes vasculares cerebrais isquêmicos, diabetes mellitus tipo 2 e alguns tipos de câncer frequentes. Cerca de 2/3 das mortes no mundo devem-se a doenças que podem ser relacionadas à obesidade (diabetes, hipertensão arterial e doenças cardiovasculares). É possível verificar-se ainda o aumento do número de obesos no mundo uma vez que segundo as estatísticas do relatório, a prevalência mundial de obesidade (índice de massa corporal maior ou igual a $30 \mathrm{~kg} / \mathrm{m}^{2}$ ) quase duplicou entre 1980 e 2008. Em 2008, 10\% dos homens $14 \%$ das mulheres do mundo (500 milhões de pessoas) eram obesos, comparados com 5\% dos homens e $8 \%$ das mulheres em 1980. Os maiores percentuais de pessoas com sobrepeso ou obesidade se registram na Região das Américas (sobrepeso 62\% em ambos os sexos, e $26 \%$ de obesos) e os mais baixos na Região do Sudeste da Ásia (sobrepeso $14 \%$ em ambos os sexos, e obesidade 3\%) ${ }^{1}$.

Pode-se afirmar infortunadamente que pouco êxito fora logrado no intuito primordial de 67 
ISSN 2179-6750

reduzir os índices de sobrepeso e obesidade no mundo desde a publicação do relatório "Estatísticas Mundiais de Saúde de 2012" ao relatório homônimo publicado pela OMS no ano de 2014. Curiosos acerca deste fracasso global na importante tarefa de reduzir tais índices cientistas, estudiosos e pesquisadores debruçaram-se em possibilidades e alternativas que mostrar-se-iam mais eficazes do que as até então adotadas em reduzir a obesidade, já considerada por muitos o "Mal do Século" 2. Destes esforços surge com clareza a concepção de que o modo mais seguro e eficaz de se combater a obesidade é combatendo-a em sua origem, a infância.

A escolha da infância enquanto foco no combate à obesidade se dá segundo Sicheri e Souza (2007) ${ }^{3}$, pelo fato de que "[...] intervenções em populações já com sobrepeso são pouco eficazes" 3 aliado ao conhecimento sintetizado por Carvalho $(2011)^{4}$ de que "É mais fácil educar as crianças a comerem bem do que reeducar adultos que já comem mal”4. Tal é a relevância da temática "obesidade infantil" no mundo que na publicação "Estatísticas Mundiais de Saúde de 2014" da Organização Mundial de Saúde em sua segunda parte, intitulada: “Temas destacados" traz como segundo destaque de relevância mundial o intitulado: "A obesidade infantil aumenta: É hora de atuar”. Acerca da temática a OMS (2014) ${ }^{5}$ reforça:

\footnotetext{
"Tradicionalmente considera-se que uma criança com excesso de peso era uma criança saudável, e geralmente era aceito o conceito de que "maior é melhor". Hoje, estas percepções estão mudando, em face de evidências de que a obesidade na infância está associada com uma vasta gama de efeitos graves e complicações de saúde e um risco aumentado de doenças prematuras."
}

Sobre as doenças prematuras e complicações de saúde relacionadas diretamente à obesidade infantil, a OMS salienta os níveis elevados de hipertensão arterial, hipercolesterolemia e diabetes tipo 2, reforça que crianças com sobrepeso estão em maior risco de se tornarem adultos com sobrepeso e traz dados alarmantes tais como que estima-se existirem em 201244 milhões (6,7\%) de crianças menores de cinco anos como obesidade ou sobrepeso em todo o mundo em contraponto à aproximadamente $5 \%$ em $1990^{5}$.

Para melhor dimensionar a problemática representada pela Obesidade e Sobrepeso no Brasil vale salientar dados estatísticos tais como: 0,8\% (1.550.993) da população brasileira apresentava obesidade grave (índice de massa corporal maior ou igual a $40 \mathrm{~kg} / \mathrm{m}^{2}$ ) representando $1,14 \%$ das mulheres e 0,44\% dos homens já em 2009 6. Fica evidente ainda o crescimento significativo do número de brasileiros com excesso de peso (IMC $\geq 25$ ), Obesidade (IMC $\geq 30$ ) e Obesidade Grave (IMC $\geq 40$ ) no país nas últimas décadas ${ }^{6,7,8,9}$. Do ano de 2006 ao ano de 2011, por exemplo, o total 
ISSN 2179-6750

de brasileiros adultos com excesso de peso passou de $42,6 \%$ a $52,5 \%$ ao passo que o total de brasileiros adultos com obesidade foi de $11,8 \%$ a $17,9 \%$, no mesmo período. Tais dados representam um aumento populacional médio de $1,28 \%$ no que se refere ao excesso de peso e de $0,77 \%$ no que tange a obesidade ${ }^{10}$. É válido salientar que desde o ano de 2012, ano em que a OMS demonstrou a nível mundial a necessidade de reduzirem-se os índices de excesso de peso e obesidade no mundo e convocou as nações à instituírem esforços neste sentido1, ao ano de 2014, ano da última publicação disponível da Vigitel, tais índices no Brasil cresceram 1,5\% e 0,5\% respectivamente. Este crescimento sugere ausência, insuficiência ou ineficácia de políticas públicas e ações a nível nacional, apesar dos alertas, publicações e estudos acreditados internacionalmente apontarem a imprescindibilidade do envolvimento das nações na mobilização global relativa ao combate ao sobrepeso e a obesidade.

Compreendido desta forma, que a obesidade é um problema crescente em todo o mundo e de grande importância para o Brasil, e que especialistas de toda parte concordam que o método mais eficaz de combatê-la é atuando na obesidade infantil, propõem-se, neste estudo, realizar revisão integrativa acerca das publicações que abordem tal temática no país, a fim de fundamentar futuras pesquisas e ações.

\section{Métodos}

A revisão integrativa inclui a análise de pesquisas relevantes que dão suporte para a tomada de decisão e a melhoria da prática clínica, possibilitando a síntese do estado do conhecimento de um determinado assunto, além de apontar lacunas do conhecimento que precisam ser preenchidas com a realização de novos estudos ${ }^{11}$. Seguindo a definição anterior, proposta por Mendes $(2008){ }^{11}$, tratase este de um estudo de revisão interativa cujo objetivo fora quantificar e analisar a produção científica nacional acerca da temática "obesidade infantil no Brasil”, de forma a resumir e agrupar o conhecimento até então produzido, delimitar lacunas do conhecimento e aferir a participação dos profissionais enfermeiros na elaboração destes estudos, em comparação às demais classes profissionais.

Deste modo, em três de abril do ano dois mil e quinze institui-se busca de publicações científicas através da Biblioteca Virtual em Saúde (BVS) ${ }^{12}$. A busca seguira o percurso e obtivera resultados, respectivamente, tais quais: $1^{\circ}$ ) Digitou-se no buscador da BVS “Obesidade Infantil" ${ }^{13}$, um sinônimo em português do descritor oficial do DeCS “Obesidade Pediátrica” obtendo-se 4.961 publicações. A escolha pelo sinônimo em lugar do descritor oficial, deveu-se ao fato de que o número de publicações utilizando-se como base o sinônimo é consideravelmente superior ao 
ISSN 2179-6750

encontrado a partir do descritor oficial. $2^{\circ}$ ) No campo denominado "Filtrar", no canto superior direito da BVS, selecionou-se em "Texto completo" a opção "Disponível” obtendo-se 2583 publicações. $3^{\circ}$ ) No campo "Limite", selecionou-se "Criança" obtendo-se 1820 publicações. $4^{\circ}$ ) No campo "País/Região, selecionou-se "Brasil" reduzindo-se o quantitativo a $68.5^{\circ}$ ) No campo "Idioma", fora selecionada a opção "Português", obtendo-se ao final do primeiro momento de busca um total de 44 publicações. $6^{\circ}$ ) Após análise mais detalhada dos artigos, foram ainda excluídos aqueles que encontravam-se indisponíveis na íntegra, os que encontravam-se repetidos na busca original e aqueles cuja temática divergia completamente à pretendida, obtendo-se um total de 33 artigos.

Para melhor compreender a natureza das publicações, fora elaborado um instrumento de coleta de dados salientando para cada um dos textos os seguintes aspectos: Título; Periódico de Publicação; Ano de Publicação; Autores; Titulação dos autores; Profissão dos autores; Abordagem metodológica; Tipo de publicação (meios utilizados para fomentar e embasar os resultados da pesquisa); Cenário de pesquisa; Objeto de pesquisa; Objetivos da pesquisa; Número de crianças participantes da pesquisa; Idade das crianças participantes; Estado em que a pesquisa fora realizada e Principais resultados encontrados. A título de atualização, no dia dezesseis de janeiro de dois mil e dezesseis fora realizada nova busca na Biblioteca Virtual em Saúde (BVS) seguindo-se estritamente o mesmo percurso metodológico e aplicando-se os mesmos critérios de inclusão e exclusão utilizados na busca inicial. Deste modo adicionaram-se à pesquisa seis novos artigos cujos dados foram posteriormente extraídos e incluídos no instrumento de coleta de dados utilizado no presente estudo. Obteve-se então, como base para o estudo, um quantitativo final de trinta e nove artigos.

Para melhor análise e compreensão do conteúdo dos artigos, foram estes subdivididos em cinco grupos, de acordo com os principais objetivos identificados. Os grupos deste modo elaborados foram: Artigos aparentemente sem relação com a obesidade infantil, mas que trazem resultados nos quais esta aparece; Artigos que pretendem aferir o estado antropométrico de determinada amostra de crianças; Artigos que buscam relacionar a obesidade infantil a possíveis causas biopsicossociais; Artigos que buscam relacionar condições negativas de saúde física e mental como consequência da obesidade infantil; e Outros artigos. O último grupo fora criado para reunir os artigos cujos objetivos não se enquadram a nenhum dos demais grupos e nem representam frequência que justifique a elaboração de um novo grupo. A partir da leitura dos artigos e da análise dos dados sintetizados no instrumento de coleta, fora possível elaborar a presente revisão. 


\section{Resultados e Discussão}

O quantitativo final dos artigos analisados consta de 39 artigos, cujos títulos foram reunidos e enumerados respeitando a ordem em que surgiram no momento da busca na base de dados. Tal disposição pode ser observada no quadro 1. A título de facilitação da leitura, os artigos serão evocados doravante de acordo com os números a que correspondem no referido quadro, em lugar de por seus títulos na íntegra.

Quadro 1. Ordem dos artigos segundo surgimento na busca inicial.

\begin{tabular}{|c|c|}
\hline $\mathrm{N}^{\mathrm{o}}$ & ítulo do artigo \\
\hline 01 & Pressão arterial elevada em crianças e sua correlação com três definições de obesidade infantil. \\
\hline 02 & Excesso de peso em crianças de pré-escolas: prevalência e fatores associados. \\
\hline 03 & Influência das variáveis nutricionais e da obesidade sobre a saúde e o metabolismo. \\
\hline 04 & Programa Bolsa Família e estado nutricional infantil: desafios estratégicos. \\
\hline 05 & $\begin{array}{l}\text { Associação entre desnutrição em crianças moradoras de favelas, estado nutricional materno e fatores } \\
\text { socioambientais. }\end{array}$ \\
\hline 06 & Avaliação do estado nutricional de crianças menores de 10 anos no município de Ferros, Minas Gerais. \\
\hline 07 & $\begin{array}{l}\text { A obesidade e sua associação com os demais fatores de risco cardiovascular em escolares de } \\
\text { Itapetininga, Brasil }\end{array}$ \\
\hline 08 & $\begin{array}{l}\text { Estado nutricional de escolares de } 1^{\mathrm{a}} \text { a } 4^{\mathrm{a}} \text { séries do Ensino Fundamental das escolas urbanas da cidade } \\
\text { de Pelotas, Rio Grande do Sul, Brasil. }\end{array}$ \\
\hline 09 & Anemia e obesidade: um paradoxo da transição nutricional brasileira \\
\hline 10 & Estado nutricional de escolares em Porto Velho, Rondônia. \\
\hline 11 & Acesso à alimentação escolar e estado nutricional de escolares no Nordeste e Sudeste do Brasil, 1997. \\
\hline 12 & Prevalência em crianças de fatores de risco para as doenças cardiovasculares \\
\hline 13 & Percepção materna e autopercepção do estado nutricional de crianças e adolescentes de escolas privadas. \\
\hline 14 & $\begin{array}{l}\text { Pobreza, desnutrição e obesidade: inter-relação de estados nutricionais de indivíduos de uma mesma } \\
\text { família. }\end{array}$ \\
\hline 15 & $\begin{array}{l}\text { Associação entre estado nutricional e atividade física em escolares da Rede Municipal de Ensino em } \\
\text { Corumbá - MS }\end{array}$ \\
\hline 16 & Obesidade, práticas alimentares e conhecimentos de nutrição em escolares. \\
\hline 17 & Obesidade infantil: como podemos s \\
\hline 18 & Relação do Índice de Desenvolvimento Humano e as Variáveis Nutricionais em Criar \\
\hline 19 & $\begin{array}{l}\text { Prevalência de sobrepeso e obesidade infantil na cidade de Feira de Santana-BA: detecção na família } \mathrm{x} \\
\text { diagnóstico clínico. }\end{array}$ \\
\hline 20 & Sobrepeso e obesidade infantil: influência de fatores biológicos e ambientais em Feira de Santana, BA. \\
\hline 21 & em crianças menores de 6 anos de idade em Florianópolis, SC \\
\hline 22 & $\begin{array}{l}\text { Prevalência de sobrepeso e fatores associados em crianças ingressantes no ensino fundamental em um } \\
\text { município da região metropolitana de São Paulo, Brasil. }\end{array}$ \\
\hline 23 & Influência da televisão na prevalência de obesidade infantil em Ponta Grossa, Paraná \\
\hline 24 & $\begin{array}{l}\text { Sobrepeso e obesidade em crianças de cinco a dez anos de idade beneficiárias do Programa Bolsa } \\
\text { Família no estado de Sergipe, Brasil. }\end{array}$ \\
\hline 25 & Panorama do estado antropométrico dos escolares brasileiros. \\
\hline 26 & Avaliação antropométrica de crianças em creches do município de Bezerros, PE. \\
\hline 27 & Avaliação e classificação do risco familiar em uma escola de educação infantil. \\
\hline 28 & Adiposidade e perfil metabólico em crianças de escolas da zona urbana de Ouro Preto-MG. \\
\hline 29 & Tradução e validação da versão brasileira do Children's Eating Attitudes Test (ChEAT). \\
\hline 30 & Magreza e sobrepeso em escolares de Rio Branco, AC, Brasil \\
\hline 31 & Desenvolvimento motor de crianças obesas \\
\hline 32 & Percepção e insatisfação corporal: um estudo em crianças bras \\
\hline 33 & Sistema de vigilância alimentar e nutricional no Estado de São Paulo, Brasil: exp \\
\hline
\end{tabular}




\begin{tabular}{|c|c|}
\hline \multicolumn{2}{|c|}{ ISSN 2179-6750 } \\
\hline & implementação e avaliação do estado nutricional de crianças. \\
\hline 34 & $\begin{array}{l}\text { Pontos de venda de alimentos e associação com sobrepeso/obesidade em escolares de Florianópolis, } \\
\text { Santa Catarina, Brasil. }\end{array}$ \\
\hline 35 & Aumento da Pressão Arterial e Obesidade na Infância: Uma Avaliação Transversal de 4.609 Escolares. \\
\hline 36 & Estado nutricional de escolares segundo a localização geográfica das escolas em Sorocaba, São Paulo. \\
\hline 37 & $\begin{array}{l}\text { Pressão arterial, crescimento alcançado e estado nutricional de crianças de seis e de dez anos de idade de } \\
\text { escolas públicas de Florianópolis, Santa Catarina. }\end{array}$ \\
\hline 38 & Obesidade infantil e uma proposta de Educação Física preventiva. \\
\hline 39 & $\begin{array}{l}\text { Estado nutricional de crianças de baixo e alto nível socioeconômico de creche filantrópica e escola } \\
\text { primária privada em Belo Horizonte-MG. }\end{array}$ \\
\hline
\end{tabular}

O primeiro grupo de análise elaborado comporta os artigos em cujo título e objetivos não há nenhuma referência clara à obesidade infantil. São estes os de número 4 e 5 . O primeiro por tratarse de um estudo direcionado a crianças beneficiárias do Bolsa Família, e portanto, em situação de vulnerabilidade social e o segundo, uma vez que traz no título a palavra "desnutrição" e nenhuma referência faz à obesidade. Entretanto, o quarto artigo traz como resultado que dentre a população pesquisada $5,2 \%$ foi classificada como obesa e o quinto artigo demonstra que $11,3 \%$ das crianças do estudo apresentavam sobrepeso/obesidade contrapondo-se a $8,6 \%$ de desnutridos.

Devido ao elevado número de artigos em cujos objetivos constava a aferição e análise do estado nutricional/antropométrico de crianças de determinada amostra, representando estes um total de $71,8 \%$ do total de artigos analisados, criou-se o segundo grupo de análise, do qual fazem parte os artigos 1, 2, 4, 5, 6, 7, 8, 10, 11, 12, 15, 16, 19, 21, 22, 23, 24, 26, 28, 29, 30 32, 33, 34, 35, 36, $37 \mathrm{e}$ 39. É válido salientar que alguns artigos apresentaram resultados referentes apenas à obesidade (desconsiderando os valores de sobrepeso), outros apresentaram valores para sobrepeso (nos quais incluíram a obesidade, mas não a diferenciaram) e outros ainda trouxeram nos resultados valores de sobrepeso e de obesidade de forma separada. Deste modo afere-se que dentre o total de crianças analisadas $6,9 \%$, em média, foram classificadas como obesas sendo o menor valor encontrado no décimo quinto artigo e correspondendo a $2,7 \%$ e o maior valor encontrado no trigésimo sétimo artigo, correspondendo à 13\%. Quanto aos valores de sobrepeso (considerando os artigos em que os valores vieram indissociados aos de obesidade e os valores obtidos através da soma dos valores referentes a estas duas variáveis, quando apresentados de forma independente nos artigos), obtevese uma média de $18,7 \%$, sendo o menor valor verificado no sexto artigo e equivalente $6,7 \%$ e o maior valor no trigésimo sétimo artigo, representando 38,1\% da amostra analisada. Quanto ainda aos valores de sobrepeso (tomados de forma separada aos de obesidade), obteve-se uma média de $13,9 \%$, estando o menor valor contido no décimo quinto artigo e equivalendo à 6,5\% e o maior valor no trigésimo sétimo artigo e equivalente à $25,1 \%$.

$\mathrm{O}$ modo de exposição iníquo dos resultados quanto à obesidade e sobrepeso nos artigos 72 
ISSN 2179-6750

analisados dificulta em muito o estudo destes, enquanto conjunto, ficando dede já a sugestão a futuros pesquisadores que elaborem seus resultados expondo separadamente valores de sobrepeso e obesidade, deixando a cargo do leitor considera-los em união ou não. É importante também, na metodologia, que fique claro quando o valor exposto para sobrepeso engloba os aferidos para obesidade e quando não engloba. Em grande parte dos artigos é necessária uma leitura muito atenciosa para chegar-se a esta conclusão o que pode dificultar a compreensão dos leitores ou mesmo induzir a erros na análise dos resultados. Independente, no entanto, do modo como os artigos expuseram os achados obtidos através das aferições nutricionais e antropométricas, é notório que os resultados referentes ao sobrepeso e a obesidade foram unanimemente alarmantes, indo de encontro aos estudos e alertas globais.

Os artigos cujos objetivos propunham delimitar causas e fatores relacionados ao estabelecimento do sobrepeso e da obesidade na infância compuseram o terceiro grupo de analise e correspondem aos artigos de número $2,11,12,13,15,16,20,22,23,34$, e 39. Através dos resultados obtidos nos referidos estudos, compreendem-se que uma série de fatores contribui positiva ou negativamente ao desenvolvimento da obesidade infantil. Far-se-á doravante o resumo dos principais fatores delimitados nos estudos e do modo como interferem nas variáveis de sobrepeso e obesidade infantil aferidas. Segundo Schuch et al (2014) ${ }^{14}$ são cinco os principais fatores detectados no decorrer de suas pesquisas com relação estatisticamente significativa às variáveis de sobrepeso e obesidade, sendo estas tais quais: o número de integrantes de uma família a residirem no mesmo domicílio, que quanto maior, menores serão as taxas de sobrepeso apresentadas pelas crianças desta família; a escolaridade das mães, uma vez que determina que filhos de mães com ensino superior têm menores prevalências de excesso de peso; a idade das mães ao nascimento dos filhos, posto que os nascidos de mães adolescentes apresentaram as menores taxas de excesso de peso; o peso ao nascer, uma vez que as crianças com baixo peso tiveram as menores taxas de obesidade infantil ao passo que as nascidas com peso superior a $4.000 \mathrm{~g}$ detiveram as maiores taxas; a idade gestacional no momento do parto, verificado que crianças prematuras apresentam menores taxas de sobrepeso quando comparadas as nascidas a termo ${ }^{14}$. Nas crianças de rede pública, o sobrepeso tende a prevalecer nas que recebem refeições gratuitas nas escolas ${ }^{15}$. Fora detectada a falta de percepção adequada do peso entre crianças e suas genitoras, sobretudo nos casos em que havia excesso de peso, o que facilita o desenvolvimento de alterações nutricionais tais quais o sobrepeso e a obesidade infantil ${ }^{16}$. As crianças com sobrepeso são menos ativas, praticam atividades físicas menos intensas e gastam mais tempo assistindo à televisão e jogando videogames, quando comparadas às eutróficas ${ }^{17}$. Crianças com menos conhecimento de nutrição e práticas 
ISSN 2179-6750

alimentares menos saudáveis apresentaram cinco vezes mais chances de serem obesas ${ }^{18}$. Fora constatada obesidade mais comumente em crianças cujos genitores possuíam níveis mais elevados de escolaridade, alta renda familiar e presença nas residências de TV, computador, telefone e vídeo game. Fora menos comum constatar-se obesidade em crianças com antecedente de repetência escolar, consumo de verduras em elevada frequência (3x/semana no mínimo) e realização sistemática de atividade física ${ }^{19}$. Corroborando com o dado supra exposto de que as altas rendas familiares contribuem positivamente no desenvolvimento da obesidade infantil, Ferreira et al (2005) afirma que crianças de alto nível socioeconômico apresentam maiores índices de obesidade e sobrepeso $^{20}$.

Corroborando com a relação entre a variável "presença de TV nas residências" e maiores índices de obesidade infantil, aferiu-se que assistir televisão diariamente por quatro ou mais horas eleva estes índices. Em consonância ao dado anterior de que práticas alimentares menos saudáveis elevam as chances de desenvolver obesidade na infância explicita-se que a frequência elevada de consumo de alimentos "não saudáveis" atua no mesmo sentido. Os dados são complementares em lugar de iguais, uma vez que compreende-se que práticas alimentares menos saudáveis incluem não apenas a ingestão elevada de alimentos "não saudáveis", mas o baixo consumo de alimentos considerados saudáveis. Mães obesas e a disponibilidade domiciliar per capita de óleo superior a 23,65ml (cerca de três colheres de sopa/dia) demonstraram relação positiva com o desenvolvimento do sobrepeso e da obesidade infantil ${ }^{21}$. Salienta-se que o consumo de bebidas industrializadas, com predominância de açúcares simples denota comportamento que predispõe ao sobrepeso ${ }^{22}$. Borges (2007) ${ }^{23}$, não apenas confirma a relação entre o ato de assistir TV por períodos prolongados e o desenvolvimento da obesidade na infância como complementa afirmando que ingerir farináceos no decorrer desta prática eleva ainda mais os índices da referida patologia ${ }^{23}$. Demonstrou-se que a obesidade infantil relaciona-se com os pontos de venda de alimentos utilizados pelos familiares das crianças, de modo a que a utilização de padarias desencadeia índices mais elevados e a utilização de supermercados os reduz. Por fim sugere-se que a ampla disponibilidade domiciliar de alimentos industrializados/ultraprocessados ricos em açúcar, gordura e calorias, por estimular o consumo alimentar em quantidade excessiva e qualidade inadequada, constitui forte fator vinculado ao aumento da prevalência de sobrepeso e obesidade infantil ${ }^{24}$. De modo geral os estudos confirmam a noção já consagrada na literatura científica do caráter multifatorial do desenvolvimento da obesidade infantil, o que reforça a necessidade da elaboração de estratégias capazes de atuar concomitantemente no enfrentamento dos amplos fatores de risco para o estabelecimento e manutenção da patologia. Fica evidente deste modo, que as ações a instituírem-se no intuito de 
ISSN 2179-6750

combater a obesidade infantil devem possuir indubitavelmente caráter multidisciplinar, de modo a envolver em todas as etapas de elaboração, execução e avaliação profissionais de diversas áreas e especialidades, além da participação da mídia, governo e população como um todo.

Artigos que buscaram relacionar condições negativas de saúde física e mental como consequência da obesidade infantil constituíram o quarto grupo de análise, sendo estes os de número $1,3,7,12,29,31,32,35,37$. Os principais resultados emergentes de tais estudos serão abordados doravante, bem como as inter-relações entre estes e análises pertinentes. A relação entre a hipertensão arterial e a obesidade infantil fora largamente estudada entre os artigos que compuseram o quarto grupo, configurando-se como principal objetivo ou um dos objetivos de pesquisa de aproximadamente $66,7 \%$. Dentre os estudos que abordaram a relação, fora unânime a conclusão de que a hipertensão configura-se como uma das possíveis consequências da obesidade infantil. Fora determinado que a obesidade infantil predispõe ao desenvolvimento de hipertensão arterial tanto na infância quanto nas fases de vida subsequentes, bem como que quanto maior o grau de obesidade na infância e o tempo de manutenção desta condição, maior será a probabilidade de desenvolver hipertensão arterial e maior a gravidade desta hipertensão, independente da fase em que se manifeste ${ }^{25,26,27,28,29,30}$. A segunda relação mais abordada entre os artigos fora entre obesidade infantil e o desenvolvimento de diabetes. Aproximadamente 33,3\% dos artigos abordaram o tema e em todos os que o fizeram fora consenso que crianças com obesidade têm maior pré-disposição ao desenvolvimento de diabetes, seja esta patologia detectada na infância ou nas demais fases da vida 26,27,28. Determinou-se que a obesidade infantil configura fator de risco para doenças cardiovasculares, disfunções metabólicas e dislipidemia tanto na infância quando nas demais fases da vida ${ }^{26,27}$. Crianças obesas apresentam dificuldades motoras com maior frequência, quando comparadas às eutróficas ${ }^{31}$. Determinou-se uma maior probabilidade de desenvolvimento de alterações e distúrbios alimentares, que se desenvolverão sobretudo na pré-adolescência e adolescência, em consequência da preocupação com o peso, desejo de tornar-se mais magro e tentativas de perder peso. Explicita-se que "[...] atitudes e comportamentos alimentares alterados estão presentes em graus alarmantes já entre os sete e os oito anos." Dentre os comportamentos alterados salientam-se: insatisfação corporal, preocupação e ações relacionadas com a prática de dietas, prática de exercícios físicos exagerados para perder peso, prática de purgas com uso de laxantes, diuréticos ou vômitos autoinduzidos e demasiada preocupação com a comida e seu conteúdo calórico ${ }^{32}$. Como possível explicação ao maior índice de distúrbios alimentares em crianças obesas, quando em comparação às eutróficas e às de baixo peso, se tem a noção de que “[...] desde crianças, os sujeitos internalizam um ideal de magreza e se sentem insatisfeitos e 
ISSN 2179-6750

descontes se não o tem" ${ }^{33}$. Como paralelo, observa-se que todas as condições negativas de saúde biopsicossociais analisadas têm como fator de risco comum a obesidade infantil. É válido salientar que "[...] a obesidade adquirida [na infância] tende a persistir na vida adulta, acompanhada de hipertensão e diabetes, notórios fatores de risco para doenças cardiovasculares" ${ }^{28}$, além de outras condições negativas abordadas nos artigos do presente estudo e outras tantas já consolidadas na literatura científica, que juntas, segundo a OMS, já em 2012 representavam a causa de cerca de 2/3 da mortes no mundo. Considerando-se que muitas das condições negativas oriundas da obesidade infantil não levam necessariamente a morte, mas são capazes de ocasionar grande sofrimento aos indivíduos que as desenvolvem, como a depressão, o isolamento social, dificuldades de relacionamento e insatisfação corporal, por exemplo, é possível mais uma vez dimensionar a problemática representada pela obesidade na infância e a imprescindibilidade de instituírem-se ações eficazes de prevenção e combate a esta condição.

O quinto e último grupo de análise, criado para reunir os artigos cujos objetivos não enquadram-se à nenhum dos demais grupos e nem representam frequência que justifique a elaboração de um novo grupo, constituiu-se dos artigos de número 9, 14, 17, 18, 25, 27, 33 e 38 . Dentre os referidos artigos dois abordaram a relação entre desnutrição e/ou anemia e obesidade, tendo ainda um deles relacionado estas variáveis à pobreza. Os resultados destes artigos apontam que tanto as condições de desnutrição/anemia quando as de obesidade estão presentes no Brasil, tendo alcançado no entanto, sobretudo devido a transição nutricional brasileira, a obesidade infantil uma expressividade superior a das demais condições. É consenso também que a tendência, caso não haja mudanças significativas nas politicas públicas de saúde nacional, é de que os índices de obesidade infantil tornem-se cada vez maiores, aumentando ainda mais a distância entre estes e os referentes à anemia/desnutrição. Os índices de obesidade mantem-se superiores aos de anemia/desnutrição, na infância, mesmo quando adicionada na análise a variável da pobreza ${ }^{34,35}$.

Houve ainda artigos que buscaram relacionar o Índice de Desenvolvimento Humano (IDH) e as variáveis nutricionais em crianças brasileiras, determinar o panorama do estado antropométrico dos escolares brasileiros, avaliar a classificação do risco familiar em uma escola de educação infantil e, relatar a experiência da implementação do Sistema de Vigilância Alimentar e Nutricional (SISVAN) no Estado de São Paulo. Quanto ao IDH conclui-se que "quanto menor o IDH maior a prevalência de obesidade" ${ }^{36}$. Quanto ao panorama do estado antropométrico dos escolares brasileiros, o estudo conclui que há redução da prevalência do déficit nutricional e aumento na ocorrência do excesso de peso, o que corrobora com os estudos anteriormente mencionados que buscaram relacionar estas duas variáveis ${ }^{37}$. Quanto à avaliação e classificação do risco familiar, o 
ISSN 2179-6750

estudo conclui que este classifica-se entre baixo e moderado tendo sido observado potencial para desenvolvimento de doenças crônicas não transmissíveis, como: hipertensão arterial, obesidade, alcoolismo, tabagismo, além de transtornos psicossociais. Vale notar que além do risco aferido da própria obesidade, há o risco da hipertensão arterial e dos transtornos psicossociais, ambos relacionáveis à obesidade ${ }^{38}$. Por fim deve-se ressaltar que apenas dois artigos possuíram como objetivo propor estratégias eficazes de prevenção e enfrentamento da obesidade infantil. Este número representa aproximadamente $5,1 \%$ do total de artigos analisados na presente revisão. Dentre estes artigos, um expôs como resultados que uma vez que os programas de intervenção ainda têm pouco consenso, a prevenção continua sendo o melhor caminho. Como principais estratégias de prevenção da obesidade destacaram-se: diminuição da ingestão calórica, prevenção do ganho excessivo de peso, aumento do gasto energético/ aumento da atividade física e incorporação ao currículo formal das escolas, em diferentes séries, do estudo de nutrição e hábitos de vida saudável, iniciando-se preferencialmente na primeira década de vida ${ }^{39}$. O outro artigo, dentre os que propuseram-se a elaborar estratégias de enfrentamento a obesidade infantil, corrobora a conclusão de que a prevenção é o modo mais eficaz de atuação, e de que hábitos alimentares saudáveis, a realização regular de atividade física e a educação em saúde nas escolas quanto à nutrição e hábitos de vida saudáveis configuram meios eficazes de realizar tal prevenção. Destaca ainda o papel central do profissional de educação física, que ao atuar nas escolas pode obter protagonismo no desenvolvimento da mencionada educação em saúde bem como colaborar de modo teórico e pratico no desenvolvimento dos hábitos saudáveis de vida na infância, criando mecanismos para que estes mantenham-se ao longo do tempo ${ }^{40}$.

Segue-se agora a exposição e analise das principais informações obtidas a partir dos itens reunidos no instrumento de coleta de dados elaborado para o presente estudo. Quanto aos periódicos de publicação os artigos dividem-se em 21 periódicos. No primeiro lugar no ranking de publicação encontram-se empatados, com 6 artigos cada, o Jornal de Pediatria e os Cadernos de Saúde Pública. Ocupam o segundo e o terceiro lugar, com 4 e 3 publicações, respectivamente a Revista Paulista de Pediatria e os Arquivos Brasileiros de Cardiologia. Em quarto lugar encontramse empatadas, com 2 publicações cada, a revista Ciência \& Saúde Coletiva, a Revista de Nutrição e a Revista Médica de Minas Gerais. Os demais 14 periódicos ocupam juntos o quinto lugar do ranking, com 1 publicação cada.

Analisando o ranking dos cinco primeiros lugares, nota-se que a temática obesidade infantil tem sido mais amplamente pesquisada e publicada por revistas da área médica (pediatria e cardiologia, sobretudo), seguidas por revistas da área de saúde pública. Nota-se também a inserção, 
ISSN 2179-6750

ainda que menos expressiva, de periódicos da área de nutrição. Vale lembrar que não há impedimento por parte dos periódicos de que autores de outras profissões ou áreas de especialização submetam artigos para publicação. É de se considerar, no entanto, que revistas de cada área temática costumam ser mais consumidas e ter maiores índices de publicação por profissionais da área comum ao periódico. Pode-se deduzir então, através da análise dos periódicos, que a temática tem representado maior interesse aos profissionais médicos, principalmente pediatras e cardiologistas. Deduz-se maior interesse, em seguida, por profissionais especializados ou de alguma feita envolvidos com a área de saúde pública, e um interesse menos expressivo, mas emergente por profissionais nutricionistas.

A inquietação quanto às profissões e áreas mais diretamente envolvidas na pesquisa do tema obesidade infantil suscitou a busca detalhada da formação de cada um dos autores. A principal fonte para tal busca fora o currículo lattes dos autores, pesquisados através da Plataforma Lattes ${ }^{41}$ do Conselho Nacional de Desenvolvimento Científico e Tecnológico (CNPq). Como fontes secundárias de pesquisa, utilizadas apenas quando o autor não possuía currículo lattes devidamente cadastrado, foram utilizadas os sites oficiais das instituições de ensino e/ou pesquisa às quais vinculavam-se os autores (universidades, por exemplo), e por fim o site de busca denominado Escavador ${ }^{42}$. Existiram ainda os autores cuja formação encontrava-se presente no próprio artigo publicado. É válido salientar que fora levada em consideração a maior titulação de cada autor apenas até o ano de publicação dos artigos analisados. Não houve autores com mais de uma formação profissional de modo a que não se fez necessário elencar critérios de escolha entre as profissões.

Deste modo, as 39 publicações denotaram um total de 177 autores. Quanto à formação profissional dos autores a Nutrição aparece em primeiro lugar, representando 37,3\% dos autores. O segundo e o terceiro lugar são ocupados por Medicina e Educação Física, representando respectivamente $28,2 \%$ e $15,8 \%$. A Enfermagem surge em quarto lugar no ranking com 7,3\% seguida de Psicologia em quinto lugar com 2,8\%. Segue-se então a Fisioterapia com 2,3\%. Representando 1,1\% encontram-se Ciências Biológicas, Matemática, Química e Farmacologia. Por fim, equivalendo a 0,6\% cada, têm-se Engenharia Química, Agronomia e Estatística. Salienta-se que os valores supramencionados, bem como os por vir, representam aproximações com uma casa decimal dos percentuais reais de cada variável. Tal escolha fora feita para tornar a leitura mais dinâmica e agradável. Tais dados confirmam a hipótese que emergira da análise dos periódicos de publicação de que os profissionais médicos tem grande representatividade no total de autores da temática pesquisada. Nota-se, no entanto, que em vez de ocupar o primeiro lugar no ranking, como supunha-se na analise anterior, estes profissionais representam a segunda colocação. Emergem 
ISSN 2179-6750

então, como pesquisadores mais numerosos da temática os profissionais de Nutrição, que em análise dos periódicos surgiam com pouca expressividade. Deduz-se que tal fato deve-se a estes profissionais publicarem mais comumente em revistas de outras áreas em lugar daquelas especializadas em Nutrição, quando o tema é a obesidade infantil. Emergem também os profissionais de Educação Física como os terceiros mais numerosos autores da temática. Deduz-se que tal qual ocorre com os profissionais Nutricionistas, a maior parte das publicações dos Educadores Físicos ocorra em periódicos não especializados na área de Educação Física. Salienta-se que os profissionais de Enfermagem surgem apenas no quarto lugar do ranking e ainda assim o total de autores enfermeiros é inferior à metade do total de autores de Educação Física, que representam a posição imediatamente a cima, e menos da quinta parte do total de autores que ocupam a primeira posição no ranking.

Tal realidade permite duas conclusões deducionais. A primeira é que os profissionais de Enfermagem não estão devidamente engajados na prevenção e combate a obesidade Infantil no Brasil. A segunda é que enfermeiros, apesar de engajarem-se adequadamente no intuito de conter este mal, não tem-se envolvido com a área de pesquisa na temática, tendo portanto um número reduzido de autores nas publicações analisadas. Para concluir-se qual das hipóteses esta correta, ou se há outra causa real para o baixo índice de autores enfermeiros da temática necessitar-se-ia de pesquisa de campo direcionada a investigação específica deste dado. Trabalhando por hora com as duas deduções realizadas, pode-se afirmar que são ambas consideravelmente preocupantes. A primeira porque se há de fato uma negligencia dos profissionais de Enfermagem quanto à Obesidade Infantil no país, isto denota desatualização ou desinteresse destes profissionais frente a um problema de saúde pública não apenas nacional, mas mundial, de cujo enfrentamento depende fortemente as melhoras nos indicadores de saúde gerais e com impacto direto nos índices de morbimortalidade da população. Acredita-se que os enfermeiros podem e devem fazer a diferença neste enfrentamento, uma vez que são estes os profissionais que acompanham os indivíduos desde o pré-natal até os cuidados pós-morte, podendo, por exemplo, ser os primeiros a detectar o risco ao sobrepeso, elaborando junto às crianças e familiares estratégias capazes de eliminar tal risco e impedindo que a criança desenvolva sobrepeso e consequentemente obesidade. Salienta-se que é indubitavelmente benéfico aos indivíduos que o cuidado ofertado seja multiprofissional, de modo a que pode e deve o enfermeiro, envolver os demais membros das equipes de saúde no combate a obesidade infantil e mesmo realizar encaminhamentos a profissionais de fora das equipes, sempre que julgar necessário. Não devem, no entanto, negligenciar de nenhuma feita à atuação própria da enfermagem, não limitando de modo algum o cuidado a encaminhamentos a outros profissionais. A 
ISSN 2179-6750

segunda dedução elaborada representa dano significativo à consolidação da enfermagem enquanto ciência, posto que caso estejam os enfermeiros atuando apenas na prática mas negligenciando as pesquisas científicas, a enfermagem perde espaço frente as demais profissões e exime-se da possibilidade de adquirir expertise no tema e fundamentar as ações e cuidados em conhecimentos produzidos, publicados e consolidados pela própria Enfermagem através de seus profissionais. Sugere-se então, que instituam-se pesquisas de campo capazes de determinar com segurança os motivos pelos quais os índices de autores enfermeiros na temática da obesidade infantil demonstram-se baixos quando confrontados aos de outras profissões. De tal conhecimento deve-se então, elaborar estratégias capazes de solucionar as problemáticas que tem dificultado ou impedido a publicação realizada por enfermeiros, conferindo a estes profissionais maior representatividade no senário científico nacional referente ao tema.

Quanto à titulação dos autores no momento da publicação dos respectivos artigos, observouse que a grande maioria havia concluído o doutorado, representando 58,2\% do total. O segundo e o terceiro maior quantitativo foram compostos por mestres e graduandos, com representatividade de $16,4 \%$ e $13 \%$ respectivamente. Profissionais graduados equivaleram a 8,5\% e àqueles com titulação de pós-doutorado representaram $7 \%$ do quantitativo total de autores. Pode-se supor que a menor participação de profissionais com pós-doutorado deve-se ao fato de serem estes profissionais os em menor número geral no mercado, quando comparados aos profissionais com as demais titulações. Nota-se que as duas titulações com maior representatividade, doutorado e mestrado respectivamente, são representados por profissionais comumente envolvidos nas esferas de pesquisa e ensino. Para os profissionais que optam por seguir carreira acadêmica, por exemplo, como professores universitários, a titulação de mestrado ou doutorado configura comumente uma exigência, quando não para o ingresso, para a progressão na carreira. Algumas instituições não educacionais, também oferecem estímulos salariais e/ou de outra natureza, para àqueles com maiores titulações, além de configurar a titulação um critério de seleção para grande parte dos concursos públicos e atuar positivamente nas análises de currículo nas instituições privadas. Para estes profissionais, além de ser exigido já no processo de obtenção do título a participação em pesquisas que podem ser, e em algumas instituições devem obrigatoriamente ser publicadas em periódicos científicos regulares, é esperado que haja maior participação científica, mesmo após a obtenção do título. Há ainda a possibilidade de obter-se financiamentos com valores variados para as pesquisas. Tais financiamentos são conferidos preferencialmente à profissionais de maior titulação e com maior participação científica comprovada, isto é, com maior número de pesquisas publicadas em periódicos científicos. Não raro, as instituições financiadoras exigem ainda, de seus 
ISSN 2179-6750

financiados, que publiquem os resultados de suas pesquisas, chegando em alguns casos a estipular um quantitativo mínimo de publicações por tempo de pesquisa, para que sejam mantidos os financiamentos. Acredita-se que o motivo pelo qual o terceiro lugar tenha sido ocupado pelos estudantes de graduação é o fato de necessitarem estes, como pré-requisito para obtenção dos diplomas, engajar-se em pesquisas junto aos orientadores para elaboração dos comumente denominados Trabalhos de Conclusão de Curso (TCC). Apesar de não ser uma exigência na graduação, há a possibilidade oportuna de que tais pesquisas sejam submetidas à publicação, explicando esta possibilidade a participação de parte dos autores graduandos. Outra possibilidade de explicação é que durante a graduação, não raro os alunos unem-se a grupos de pesquisa e extensão universitária e estes grupos, devidamente orientados por um ou mais professores, produzem pesquisas e elaboram artigos para publicar os resultados obtidos. Artigos deste modo produzidos responderiam por outra parcela dos autores graduandos.

Muito preocupante, no entanto, é a pouca participação dos profissionais graduados e sem títulos posteriores. Através de tal dado elenca-se a possibilidade de que tais profissionais, em posse de seus diplomas, engajam-se no mercado de trabalho e abdicam da participação nos meios científicos. Sabe-se que a pesquisa, o que inclui àquela que emerge da prática profissional e das inquietações dela advindas, é fundamental à consolidação do saber e do caráter científico de qualquer profissão. Acredita-se que a atuação ideal é aquela na qual os profissionais não apenas buscam nos conhecimentos científicos a fundamentação para a atuação, mas encontram na atuação inquietações e vieses de pesquisa que culminem na realização, por estes profissionais, de novos artigos e publicações que retroalimentarão a base na qual estes e os demais, fundamentam as ações comuns às profissões. Deduz-se que, por ocuparem os graduados sem titulação posterior o penúltimo lugar no ranking, equivalendo a um índice inferior a sexta parte do equivalente a primeira colocação, estes profissionais não estão em consonância com a atuação adequada exposta anteriormente, isto é, não estão utilizando-se e retroalimentando o conhecimento científico como poderiam. Se for comprovada tal dedução, há de se considerar o enorme prejuízo que este dado representa à produção científica de um modo geral, uma vez uma grande parcela dos profissionais brasileiros não possuem titulações de mestrado, doutorado ou pós-doutorado. Dentre os autores enfermeiros não houve nenhum com pós-doutorado. Àqueles com doutorado ocuparam o maior índice, representando $46,1 \%$ e os graduados sem titulação posterior tiveram a menor participação, equivalendo a $7,7 \%$ do total de autores enfermeiros. Tais resultados corroboram a tendência verificada anteriormente para o conjunto total de autores dos artigos analisados.

Quanto ao ano de publicação, como visto na metodologia, não fora utilizado como critério 81 
ISSN 2179-6750

de inclusão ou exclusão o ano de publicação obtendo-se assim todos os artigos publicados até a data da pesquisa. Deste modo, compuseram o total de artigos àqueles desde o ano de 2003 até o ano de 2014, havendo para cada um dos anos neste intervalo, o mínimo de um artigo publicado. O ano com maior número de publicações fora o de 2007 , representando $20,5 \%$ do total e os de menores índices foram os anos de 2004, 2006, 2009 e 2013, empatados na última posição e representando 2,6\% do total, cada. Não fora possível neste momento elaborar relação causal entre os anos de maior publicação e os eventos a nível nacional ou global referentes ao combate à obesidade infantil. As variáveis denotam certa aleatoriedade. Acredita-se que este fato justifique-se pela possibilidade de as publicações atenderem as demandas dos autores, em lugar de surgirem em resposta às tendências e demandas nacionais e internacionais de saúde. Ou seja, deduz-se que os autores elaboraram pesquisas e consequentemente as publicam quando necessitaram destas publicações e aderiram ao tema por possuir maior afinidade pessoal ou maior facilidade de pesquisa, não em resposta às evidências e necessidades globais imediatas. Esta hipótese justificaria o fato, por exemplo, de um dos anos com o menor índice de publicações ser justamente 2013, ano posterior à publicação da OMS elencando como alerta a necessidade mundial de combate a obesidade. Apesar de no ano de 2014, dois anos após o alerta inicial e ano de novo alerta da OMS, desta vez especificamente acerca da obesidade infantil, haver um acréscimo de 10,2\% em relação às produções do ano de 2013, o ano de 2008 iguala e os anos de 2010 e 2007 superam os índices de publicação de 2014. Sugere-se pois, como tema de pesquisas futuras a relação entre os anos de maior publicação nacional direcionada a obesidade infantil e os eventos nacionais e globais acerca da temática.

Quando à abordagem metodológica, os artigos foram classificados como de abordagem quantitativa, qualitativa ou quanti-qualitativa. Àqueles de abordagem quantitativa obtiveram índice expressivamente superior aos de demais métodos, sendo seu total superior mesmo à soma dos valores totais das demais abordagens metodológicas. Deste modo temos a abordagem quantitativa representando $79,5 \%$ do total de publicações, e as abordagens qualitativas e quanti-qualitativas representando 12,8\% e 7,7\%, respectivamente. Em consonância a este dado percebeu-se, durante a leitura dos artigos, que a maior parte destes tinha como principal objetivo, ou um dos principais, aferir as características antropométricas de dada população, de modo a que os autores optaram utilizar para a realização de tal tarefa, a abordagem quantitativa.

Notou-se também que apenas uma parcela diminuta dos artigos possuía como objetivo principal ou um dos principais, a abordagem e exploração de soluções factíveis ao combate e prevenção do obesidade infantil. Este fora o principal grupo de artigos a utilizar-se da abordagem qualitativa e somou-se a ele uma parcela dos artigos de revisão de literatura cujos autores 
ISSN 2179-6750

realizaram a mesma opção metodológica. Ainda mais raros foram os autores em cujos artigos houve a proposta de aferir quantitativamente informações acerca da temática e concomitantemente analisar estas e outras informações sintetizadas e recolhidas no decorrer dos estudos de maneira qualitativa. Estes compuseram o menor grupo, optando pela abordagem quanti-qualitativa.

Quanto aos tipos de publicação, os artigos foram divididos em artigos de revisão de literatura e artigos originais. Considerou-se de revisão aqueles que limitaram-se a busca e analise de dados pré-existentes, estando estes contidos em artigos científicos ou demais fontes, como dados de pesquisa publicados em fontes oficiais ou de acreditação comprovada como por exemplo os do Sistema Nacional de Vigilância Alimentar e Nutricional (SISVAN), e do Departamento de Informática do Sistema Único de Saúde (DATASUS). Foram considerados estudos originais aqueles cujos autores foram a campo coletar dados acerca de dada população, relacionando-os direta ou indiretamente a temática do presente estudo. Deste modo aferiu-se que 77,9\% dos artigos foram estudos originais ao passo que $23,1 \%$ representavam revisão de literatura. Evidencia-se com este dado uma carência em revisões de literatura quanto à temática da obesidade infantil em âmbito nacional. Uma vez compreendido que o papel fundamental das revisões de literatura é sintetizar o conhecimento científico produzido até certo momento acerca de dada temática, compreende-se que a carência deste tipo de pesquisa teve como um dos resultados um quantitativo significativo de pesquisas originais com objetivos similares, se não iguais, variando por vezes, apenas a população a ser estudada. Com mais artigos de revisão, novos autores poderiam partir do conhecimento já reunido em estudos originais anteriores e delimitariam objetivos novos, levando o conhecimento científico a evoluir gradativamente, em lugar de reproduzirem-se em diferentes populações e regiões do país, pesquisas com objetivos similares que têm obtido sistematicamente, resultados equivalentes.

Dentre os artigos cujos autores foram a campo coletar dados, em 64,1\% o campo de coleta escolhido foram escolas, entre as de rede pública e privada. Entre os demais cenários, constam residências em comunidades carentes, residências de beneficiários de programas de auxílio do governo federal, ambulatórios e postos de saúde. Aliando-se o fato de que 79,5\% dos artigos estudados foram classificados como quantitativos e que a expressiva maioria dos autores escolhera as escolas como campo de coleta de dados, pode-se inferir que parte da escolha dos cenários devese ao fato de haver nestes, concentração abundante de crianças em horários estabelecidos, o que atuaria como facilitador ao processo de pesquisa.

Quanto aos estados onde ocorreram as pesquisas, foram considerados àqueles onde localizaram-se os campos de coleta de dados, nos estudos originais e àqueles aos quais prestavam 
ISSN 2179-6750

referência os dados coletados em fontes tais qual o SISVAN e o DATASUS, no caso de revisões de literatura. Foram desconsiderados àqueles cujos dados não direcionavam-se a estados determinados, os nos quais tal informação não estivera disponível e àqueles de conteúdo estritamente teórico e qualitativo, nos quais a obesidade infantil fora abordada de forma ampla, não atendo-se a populações restritas a estados. Salienta-se deste modo que obteve-se um total de 29 artigos para este grupo analítico, dados os critérios de inclusão e exclusão mencionados. Surgiram então, como estados em cujo território desenvolveram-se pesquisas publicadas acerca do tema, 15 estados. O primeiro lugar no ranking entre os estados fora assumido por São Paulo, retratado em 17,2\% dos artigos. Em segundo lugar encontraram-se empatados Santa Catarina e Minas Gerais, ambos representando 13,8\% dos estudos. O terceiro lugar fora ocupado pelo Rio Grande Sul com 10,3\%, seguido pelo Paraná e Maranhão empatados na quarta posição com 6,9\% cada. Ocupando a quinta posição, com 3,4\% cada, seguiram empatados os estados do Espírito Santo, Alagoas, Mato Grosso do Sul, Bahia, Sergipe, Pernambuco, Acre, Rondônia e Rio de Janeiro. Considerando-se que há no Brasil 27 unidades federativas, sendo 26 estados e um distrito federal e que destas tão somente 15 foram estudadas, o que equivale a 55,6\% do total de estados, infere-se que grande parte do território brasileiro encontra-se ainda sem nenhum tipo de estudo em caráter científico publicado acerca da obesidade infantil aumentando a vulnerabilidade da população deste território, uma vez que há menor quantitativo de informações que atendam as especificidades do mesmo. Deve-se salientar ainda que do total de estados pesquisados, 55,1\% dos artigos detiveram-se a apenas quatro estados, sendo estes: São Paulo, Santa Catarina, Minas Gerais e Rio Grande do Sul. Conclui-se então que além de apenas pouco mais da metade dos estados brasileiros configurarem campo de estudo para artigos científicos publicados, há uma forte concentração de publicações entre os estados pesquisados, possibilitando que haja mesmo entre estes, grandes parcelas da população sem nenhum estudo acerca de si no tocante a obesidade infantil. Dado o senário epidemiológico mundial, a urgência em prevenir e combater a obesidade infantil e considerando-se a grande diversidade sociocultural do Brasil, este déficit de estudos no território nacional, delimitando grandes parcelas da população não estudadas, somado ao subsequente déficit no conhecimento específico acerca da temática expõem a população a maior vulnerabilidade quando a obesidade infantil e as doenças a ela relacionadas configurando-se em fator dificultador à implementação de políticas públicas eficazes no intuito de garantir saúde e qualidade de vida à população.

Quanto ao número de crianças estudadas nas publicações, somando-se todos os estudos obteve-se o total de 1.150 .342 crianças. O estudo com menor número de crianças abarcou 347 delas e o com maior número 1.007.696 sendo estes os de número 33 e 32 respectivamente, de acordo com 
ISSN 2179-6750

o quadro 1. Estudos com amostras inferiores a 1.000 crianças representaram 44,4\% dos estudos.

Àqueles com amostras entre 1.000 e 5.000, corresponderam a 40,7\%. Os situados entre 10.000 e 80.000 a $11,1 \%$ e os com amostras superiores a 1.000 .000 de crianças somaram 3,7\% dos artigos. Nota-se que os estudos tendem a diminuir à medida que elevam-se as amostras de crianças. Atribuise a este fato o aumento da complexidade e das dificuldades de pesquisa na medida em que elevamse as amostras pesquisadas. Quanto à idade dos pesquisados, esta variou entre zero e dezenove anos, sendo designado pelos autores a faixa etária que pretenderam estudar e variando esta significativamente de estudo a estudo tornando a formulação de grupos analíticos pouco elucidativa, uma vez que cada grupo representaria ínfima relevância estatística. Salienta-se ainda que poucos foram os autores que justificaram no decorrer do artigo a escolha das faixas etárias estudadas. Infere-se que estas foram escolhidas, por aqueles que não apresentaram justificativa, de acordo com critérios de maior facilidade ao desenvolvimento da pesquisa de acordo com as possibilidades de cada autor ou grupo de autores.

\section{Conclusão}

Todos os artigos confirmaram incontestavelmente que a obesidade é uma realidade significativa e crescente nas diferentes regiões estudadas do Brasil, o que vai de encontro às afirmações da OMS nas Estatísticas Mundiais de Saúde de 2014 e corrobora com a necessidade de instituírem-se novos e mais aprofundados estudos acerca da temática em áreas pouco ou ainda não estudadas do país.

Os artigos que propuseram relacionar a obesidade a condições biopsicossociais negativas, obtiveram sem exceção resultados que comprovam os inúmeros malefícios da obesidade à saúde da criança.

Os profissionais de Nutrição são os que mais pesquisam e publicam acerca do tema. A participação dos enfermeiros é ainda bem pequena, e ainda menor a de enfermeiros graduados sem titulação posterior. Esta realidade denota necessidade de maior engajamento desta classe profissional nas pesquisas relacionadas à obesidade infantil.

Tal patologia, além da grande representatividade mundial, vem alcançando níveis alarmantes no Brasil e é um fator predisponente a inúmeras patologias, dentre as quais destacam-se as de grande índice de morbimortalidade, como Infarto Agudo do Miocárdio, Acidente Vascular Cerebral, Diabetes Mellitos tipo 2 e Cânceres como o de mama, o que torna indispensável o maior envolvimento da enfermagem no combate a este mal.

A maior parte das pesquisas foi quantitativa e realizada nas escolas, o que indica que o foco 85 
ISSN 2179-6750

dos pesquisadores até o momento tem sido dimensionar o problema, aferindo seus números nas populações pesquisadas para posteriormente melhor conhecerem causas e capacitarem-se para pesquisar e propor soluções. Dentre àqueles que buscaram determinar causas e fatores predisponentes, as publicações confirmaram o caráter multifatorial do desenvolvimento da obesidade na infância, perpassando os âmbitos biológico, psíquico, social e os fatores ambientais envolvidos. Raros foram os autores cujas pesquisas buscaram como objetivo central determinar mecanismos eficazes de combate e prevenção à obesidade na infância, e dentre os que propuseramse a tal, nenhum propôs estratégias originais, limitando-se a rever e sintetizar estratégias publicadas anteriormente na literatura. A escolha das escolas justifica-se pelo quantitativo de crianças em horários pré-determinados, o que atuaria como facilitador no processo de pesquisa.

Julga-se, pois, importante que novos estudos sejam realizados em caráter quanti-qualitativo em escolas ou outros campos com suficiente número de crianças, prioritariamente nos estados e municípios com pouca ou nenhuma publicação acerca do tema. Considera-se de suma importância que tais trabalhos envolvam não somente as crianças, mas os familiares e professores uma vez que a transmissão de hábitos alimentares e de vida saudáveis dependem em muito da atuação destes. Compreendida a relevância dos profissionais de enfermagem no cuidado direto a população e a continuidade deste cuidado no decorrer da vida dos indivíduos cuidados, bem como a possibilidade factível do estabelecimento de alianças entre estes profissionais, as escolas, famílias e demais instâncias e instituições da comunidade, sugere-se fortemente que sejam enfermeiros a realizarem parte dos estudos por vir. Posto a confirmação sistemática do caráter multifatorial das causas da obesidade infantil e da multiplicidade das patologias e agravos a ela relacionados, sugere-se que os novos estudos dediquem-se prioritariamente a propor soluções de combate e prevenção a este mal, em lugar de aterem-se a quantifica-lo e caracteriza-lo quanto a causas e efeitos. Tais achados serviriam indubitavelmente para fundamentar as ações de enfermagem e demais profissionais no enfrentamento ao já considerado mal do século.

\section{Referências}

1. World Health Organization, 2012. Word Health Statistics 2012. Parte II. 34-36. [Acesso em 02 de set 2015]. Disponível em: http://www.who.int

2. Lima RM. Obesidade: o mal do século. Rev. Persp. Online, 2013. Campos dos Goytacazes, $1(2): 86-99$.

3. Sichieri R, Souza RAG. Epidemiologia da Obesidade. In: Kac G, Sichieri R, Gigante DP Epidemiologia nutricional. Rio de Janeiro: Ed. Fiocruz/Atheneu; 2007. 347-358. 
ISSN 2179-6750

4. Universidade de Brasília [homepage na internet]. Pesquisa mostra aumento da obesidade no Brasil [Acesso em 18 de set de 2015]. Disponível em: http://unb.br

5. World Health Organization, 2014. Word Health Statistics 2014. Parte II. 40-41[Acesso em 05 de set de 2015]. Disponível em: http://www.who.int

6. Instituto Brasileiro de Estatística e Geografia [homepage na internet]. Pesquisa de Orçamentos Familiares 2008-2009 [Acesso em 02 de fev]. Disponível em: http://www.ibge.gov.br

7. Instituto Brasileiro de Estatística e Geografia [homepage na internet]. Pesquisa de Orçamentos Familiares 2002-2003[Acesso em 02 de fev]. Disponível em: http://www.ibge.gov.br

8. Brasil. Ministério da Saúde. Departamento de Atenção básica. Pesquisa Nacional sobre Saúde e Nutrição (PNSN) 1989. [Acesso em 11 de set de 2015]. Disponível em: http://dab.saude.gov.br/portaldab

9. Instituto Brasileiro de Estatística e Geografia. Estudo Nacional de Despesa Familiar (ENDEF) 1974-75. [Acesso em 11 de set 2015]. Disponível em: http://biblioteca.ibge.gov.br 10. Ministério da Saúde. Secretaria de Vigilância em Saúde. Departamento de Vigilância de Doenças e Agravos não Transmissíveis e Promoção da Saúde. Vigitel Brasil 2014: vigilância de fatores de risco e proteção para doenças crônicas por inquérito telefônico. Brasília: Ed. Ministério da Saúde, 2015.

11. Mendes KDS, Silveira RCCP, Galvão CM. Revisão integrativa: método de pesquisa para a incorporação de evidências na saúde e na enfermagem. Texto contexto - enferm. [Online]. 2008; 17(4):758-764.

12. Biblioteca Virtual em Saúde (BVS). [Acesso em 03 de abr de 2015]. Disponível em: http://www.bireme.br

13. Descritores em Ciências da Saúde(DeCS). [Acesso em 03 de abr de 2015]. Disponível em: http://decs.bvs.br

14. Schuch I, Castro TG, Vasconcelos FAG, Dutra CLC, Goldani MZ. Excesso de peso em crianças de pré-escolas: prevalência e fatores associados. J. Pediatr. Rio de Janeiro [Online]. 2013; 89(2):179-188.

15. Burlandy L, Anjos LA. Acesso à alimentação escolar e estado nutricional de escolares no Nordeste e Sudeste do Brasil, 1997. Cad. Saúde Pública [Online]. 2007; 23(5):1217-1226.

16. Boa-Sorte N, Neri LA, Leite MEQ, Brito SM, Meirelles A R, Luduvice FBS et al. Percepção materna e autopercepção do estado nutricional de crianças e adolescentes de escolas 87 
ISSN 2179-6750

privadas. J. Pediatr. Rio de Janeiro [Online]. 2007; 83(4):349-356.

17. Baruki SBS, Rosado LEFPL, Rosado GP, Ribeiro RCL. Associação entre estado nutricional e atividade física em escolares da Rede Municipal de Ensino em Corumbá - MS. Rev Bras Med Esporte [Online]. 2006; 12(2):90-94.

18. Triches RM, Giugliani ERJ. Obesidade, práticas alimentares e conhecimentos de nutrição em escolares. Rev. Saúde Pública, São Paulo [Online]. 2005; 39(4): 541-547.

19. Oliveira AMA, Cerqueira EMM, Souza JS, Oliveira AC. Sobrepeso e obesidade infantil: influência de fatores biológicos e ambientais em Feira de Santana, BA. Arq Bras Endocrinol Metab [Online]. 2003; 47(2):144-150.

20. Ferreira QTM, Queiroz AM; Ramos PS et al. Estado nutricional de crianças de baixo e alto nível socioeconômico de creche filantrópica e escola primária privada em Belo HorizonteMG. Rev Med Minas Gerais. 2005;15(1):6-10.

21. Mondini L, Levy RB, Saldiva SRDM, Venâncio SI, Aguiar JA, Stefanini MLR. Prevalência de sobrepeso e fatores associados em crianças ingressantes no ensino fundamental em um município da região metropolitana de São Paulo, Brasil. Cad. Saúde Pública [Online] 2007. 23(8):1825-1834.

22. Gama SR, Carvalho MS, Chaves CRMM. Prevalência em Crianças de Fatores de risco como Pará Doenças Cardiovasculares. Cad. Saúde Pública [Online] Rio de Janeiro, 2007. 23(9): 2239-2245.

23. Borges RB et al. Influência da televisão na prevalência de obesidade infantil em Ponta Grossa, Paraná. Ciência, Cuidado e Saúde. 2007 [Acesso em 03 fev 2015]. Disponível em: http://www.periodicos.uem.br.

24. Motter AF et al. Pontos de venda de alimentos e associação com sobrepeso/obesidade em escolares de Florianópolis, Santa Catarina, Brasil. Cad. Saúde Pública, Rio de Janeiro, 2015. 31(3):620-632.

25. Moraes LL et al. Pressão arterial elevada em crianças e sua correlação com três definições de obesidade infantil. Arq Bras Cardiol. São Paulo, 2014.102(2):175-180.

26. Azevedo FR, Brito BC. Influência das variáveis nutricionais e da obesidade sobre a saúde e o metabolismo. Rev. Assoc. Med. Bras.São Paulo, 2012. 58(6):714-723.

27. Pereira Abel et al. A obesidade é Sua Associação com os demais Fatores de risco cardiovascular em Escolares de Itapetininga, Brasil. Arq. Bras. Cardiol. São Paulo, 2009. 93(3):253-260. 
ISSN 2179-6750

28. Gama SR, Carvalho MS, Chaves CRMM. Prevalência em Crianças de Fatores de risco como Pará Doenças Cardiovasculares. Cad. Saúde Pública, Rio de Janeiro, 2007. 23(9):22392245. Disponível em: http://www.scielo.br

29. Rosaneli, CF et al. Aumento da Pressão Arterial e Obesidade na Infância: Uma Avaliação Transversal de 4.609 Escolares. Arq. Bras. Cardiol. São Paulo, 2014.103(3):238-244.

30. Cardoso, JL. Pressão arterial, crescimento alcançado e estado nutricional de crianças de seis e de dez anos de idade de escolas públicas de Florianópolis, Santa Catarina. [Tese Doutorado em Saúde Materno Infantil] - Faculdade de Saúde Pública, Universidade de São Paulo, São Paulo, 2014.

31. Poeta LS et al. Desenvolvimento motor de crianças obesas. R. bras. Ci. e Mov 2010;18(4):18-25. [Acesso em 10 de fev 2016]. Disponível em: http://portalrevistas.ucb.br

32. Pinheiro N, Jiménez M. Tradução e validação da versão brasileira do Children's Eating Attitudes Test (ChEAT). Psicol. argum; 2012. 30(70):515-524.

33. Pinheiro N, Jiménez M. Percepção e insatisfação corporal: um estudo em crianças brasileiras. Rev Psico. 2010. 41(4):510-516.

34. Batista Filho $\mathrm{M}$ et al. Anemia e obesidade: um paradoxo da transição nutricional brasileira. Cad. Saúde Pública, Rio de Janeiro. 2008.24(supl.2):247-257.

35. Martins IS et al. Pobreza, desnutrição e obesidade: inter-relação de estados nutricionais de indivíduos de uma mesma família. Ciênc. saúde coletiva, Rio de Janeiro, 2007. 12(6) 1553 1565.

36. Jacinto-Rego SAS et al. Relação do Índice de Desenvolvimento Humano e as Variáveis nutricionais em Crianças do Brasil. Rev. salud pública, Bogotá. 2008.10(1):62-70.

37. Reis CEG, Vasconcelos IAL, Oliveira MOV. Panorama do estado antropométrico dos Escolares Brasileiros. Rev. Paulo. pediatr. São Paulo, 2011. 29(1):108-116.

38. Santos SM. Avaliação e classificação do risco familiar em uma escola de educação infantil. Revista de Pesquisa: Cuidado é Fundamental [Online] 2014. [Acesso em 19 de jan 2016]. Disponível em: http://www.seer.unirio.br.

39. Mello ED, Luft VC, Meyer F. Obesidade infantil: como podemos ser eficazes ? J. Pediatr. Rio J.), Porto Alegre, 2004. 80(3):173-182. [Acesso em 19 de jan 2016]. Disponível em: http://www.scielo.br.

40. Santos AL, Carvalho AL, Júnior JRG. Obesidade infantil e uma proposta de Educação Física preventiva. Motriz, Rio Claro, 2007. 13(3):203-213.

41. Plataforma Lattes [Acesso em 9 de fev de 2016. Disponível em: http://lattes.cnpq.br . 
ISSN 2179-6750

42. Escavador [Acesso em 9 de fev de 2016]. Disponível em: http://www.escavador.com . 